\title{
STOP TALKING! Inhibition of speech is affected by word frequency and dysfunctional impulsivity
}

\author{
Wery P. M. van den Wildenberg ${ }^{1 *}$ and Ingrid K. Christoffels ${ }^{2}$ \\ Amsterdam Center for the Study of Adaptive Control in Brain and Behavior (Acacia), Department of Psychology, University of Amsterdam, Amsterdam, Netherlands \\ 2 Leiden Institute for Psychological Research and Leiden Institute for Brain and Cognition, Leiden University, Leiden, Netherlands
}

\section{Edited by}

Diane Pecher, Erasmus University

Rotterdam, Netherlands

\section{Reviewed by}

F.-Xavier Alario, Centre National de la

Recherche Scientifique and

Aix-Marseille Université, France

Sharon Morein-Zamir, University of

Cambridge, UK

*Correspondence:

Wery P. M. van den Wildenberg,

Department of Psychology, University

of Amsterdam, Roetersstraat 15, 1018

WB, Amsterdam, Netherlands.

e-mail:w.p.m.vandenwildenberg@uva.nl
Speaking is a complex natural behavior that most people master very well. Nevertheless, systematic investigation of the factors that affect adaptive control over speech production is relatively scarce. The present experiments quantified and compared inhibitory control over manual and verbal responses using the stop-signal paradigm. In tasks with only two response alternatives, verbal expressions were slower than manual responses, but the stopping latencies of hand and verbal actions were comparable. When engaged in a standard picture-naming task using a large set of pictures, verbal stopping latencies were considerably prolonged. Interestingly, stopping was slower for naming words that are less frequently used compared to words that are used more frequently. These results indicate that adaptive action control over speech production is affected by lexical processing. This notion is compatible with current theories on speech self-monitoring. Finally, stopping latencies varied with individual differences in impulsivity, indicating that specifically dysfunctional impulsivity, and not functional impulsivity, is associated with slower verbal stopping.

Keywords: response inhibition, stop task, cognitive control, word frequency, word production, picture naming

\section{INTRODUCTION}

\section{INHIBITORY CONTROL OVER SPEECH}

On average, we produce a few thousand words a day. This uniquely human ability to communicate by speech is characterized by a profound ease and automaticity. Despite high articulation rates of about $120-150$ words per minute, error rates in speech production are down to 1 per 1000 words (Levelt, 1992). The apparent high level of adaptive control over speech production is also underscored by peoples' ability to alter the course of a sentence or even stop in the middle of word production according to an internal (re)evaluation of the situation or in response to sudden relevant changes in the external environment. For example, during a lively conversation with an accomplice about organizing a surprise party, one is able to stop talking abruptly when the birthday boy suddenly enters the room. Notwithstanding the common nature and efficiency of speech, surprisingly little work has been done to specify cognitive variables that modulate adaptive stopping control over speech production. An early study by Ladefoged et al., (1973) tested the interruptibility of speech by asking four participants to repeat a memorized short phrase. In addition, participants were instructed to stop their speech immediately upon hearing a brief and unexpected stop tone and say /ps/ instead. As long as the stop stimulus occurred within the phrase, participants could interrupt themselves equally well at virtually any point, producing the /s/ of the $/ \mathrm{ps} /$ in about $300 \mathrm{~ms}$ after presentation of the stop tone. This finding underscores the ability to stop talking at almost any point in the speech production process.

Since the descriptive work by Ladefoged et al. (1973), a more formal reaction time (RT) paradigm emerged to study stopping, or inhibitory action control, in the laboratory; the stop paradigm (Logan and Cowan, 1984; for a review see Verbruggen and Logan,
2008). A typical stop task requires participants to issue a discriminative manual response to visual go stimuli (e.g., to press a response button with the right hand to an "X" and to press a button with the left hand to an "O". During this so called go task, a visual or auditory stop-signal is presented unexpectedly on some trials, upon which participants should try to inhibit their overt response to the go signal. Given the assumptions of a formal race model, it is possible to estimate the latency of the stop process, or stop-signal reaction time (SSRT; Logan, 1994; Band et al., 2003). The SSRT reflects the RT of the internal response to the stop-signal, from stop-signal detection to the implementation of stopping, and as such provides advantages over other experimental procedures that assess response inhibition, such as versions of the go/nogo task (Logan and Cowan, 1984; see also Band et al., 2003).

The vast majority of the stop-signal literature addresses inhibitory control over binary hand or eye movements (for a review see Verbruggen and Logan, 2008). A relevant exception is presented by a recent study by Xue et al. (2008) who used the stop-signal paradigm to investigate inhibitory control over the production of speech. They compared the neural correlates of stopping manual and verbal responses using functional magnetic resonance imaging (fMRI). In the standard manual condition, participants responded with the right index and middle fingers to pictures of the letters " $T$ " and " $D$ ". They attempted to stop their response when an occasional auditory stop-signal was presented. In addition to this manual condition, a verbal letter naming condition was included that required participants to name " $T$ " or " $D$ " aloud. The behavioral results indicated that manual and verbal RT were comparable in terms of speed, but stopping was faster for letter naming responses. In addition, stopping latencies in the speech and manual inhibition tasks were positively correlated across subjects. This correlation, together with 
the observed common neural activation pattern that included the right inferior frontal cortex and pre-supplementary area, led Xue et al. (2008) to suggest a shared neural mechanism for inhibiting both manual responses and speech production.

The first aim of the present study is to further investigate whether response modality affects stopping proficiency by comparing SSRT for manual and verbal choice responses. Experiment 1 directly compares inhibitory control in these two response modalities in a two-choice setup. The design of Experiment 1 was very similar to the binary-choice setup that is commonly reported in the standard stop-signal literature. Participants were presented with a pair of simple line-drawing pictures of a tree and a door. In the manual task, the pictures called for a button press with the left or right index fingers, whereas the pictures were to be named aloud in the verbal task. Occasional presentation of a visual stop-signal allowed a straightforward comparison of stopping latencies for binary manual and verbal responses to discriminate between two pictures. Given that a wide range of responses can be stopped in about 200 ms (Logan, 1994), we hypothesize that stopping latencies are comparable for verbal and manual response modalities, predicting that both manual and verbal choice responses can be stopped in about $200 \mathrm{~ms}$.

\section{STOPPING AND THE NUMBER OF RESPONSE ALTERNATIVES}

The vast majority of the stop-signal literature reports on the efficiency of inhibitory action control over binary motor actions, like pressing a response button to the letters "X" and "O". Extending the stop-signal task from the manual to the verbal domain enables the introduction of multiple response alternatives, a setup that goes beyond the two-choice configuration that is commonly used. For example, Xue et al. (2008) also added a reading condition in which pseudo words (like "haxp") were read out loud. Pseudo word reading is more complex and turned out to be slower than binary (" $\mathrm{D}$ " vs. "T") letter naming. Analyses of SSRT indicated that stopping was also prolonged for reading one out of multiple pseudo words compared to binary letter naming.

The second aim of the present study is to test whether the number of response alternatives affects inhibitory control over verbal word production. Hence, Experiment 2 extended the binarychoice context of Experiment 1 by introducing stop-signals in a naming task with multiple pictures (Glaser, 1992). Picture naming is commonly used in speech production research because it entails all core processes of speech production, unlike verbalizing letters or pseudo words (e.g., Xue et al., 2008). Reading may proceed by mere grapheme-to-phoneme conversion (Indefrey and Levelt, 2004), whereas pseudo word reading necessarily employs grapheme-to-phoneme conversion since there are no lexical entries. In addition, and compared to standard binary stop tasks, picture naming is interesting in terms of its ecological validity. It offers a virtually unlimited set of natural stimulus-response combinations unlike response repertoires that are either limited (e.g., responding in a binary fashion) or arbitrary (e.g., pressing buttons to symbolic stimuli). Current theoretical models of word production in picture-naming tasks assume distinct cognitive processing stages that include object recognition, non-verbal conceptual processing, lexical retrieval, and articulation (e.g., Warren and Morton, 1982; Griffin and Bock, 1998; Levelt, 1999; Graves et al., 2007).
Given previous accounts of prolonged SSRT with increasing demands on response selection processes (e.g., Logan et al., 1984; Szmalec et al., 2009), we predict slower stopping when the number of verbal response alternatives is increased (Experiment 2) compared to stopping in a binary choice-naming task (Experiment 1).

\section{MANIPULATING THE RELATIVE FREQUENCY OF PICTURE NAMES}

The third aim is to test whether increasing the demands on linguistic processing affects inhibitory control over verbal responses. To this end, we introduced relative word frequency as experimental factor affecting verbal stopping latency (Experiment 2). Word frequency is a common experimental manipulation in linguistic research and indicates the degree of use of a given word, generally on the basis of counts of written corpora (Oldfield and Wingfield, 1965). In general, picture-naming latencies decrease as name frequency increases (Jescheniak and Levelt, 1994; Barry et al., 1997; Alario et al., 2004). Word frequency is generally thought to modulate resources required for lexical access (e.g., Almeida et al., 2007; Graves et al., 2007). More specifically, Ferreira and Pashler (2002) presented clear experimental evidence derived from the psychological refractory period paradigm that word frequency affects a central processing stage (see also Dent et al., 2008). If producing low-frequency words is indeed associated with an increased load on central processing, and inhibitory control requires the same central processes, then stopping is expected to be prolonged for naming less frequent compared to highly frequent words (Experiment 2).

\section{INDIVIDUAL DIFFERENCES IN IMPULSIVITY PREDICT INHIBITORY PROFICIENCY}

The task manipulations described above (i.e., response modality, the number of response alternatives, and relative word frequency) all represent variables that might affect stopping latency between experimental conditions. Another approach toward understanding factors that influence the proficiency of inhibitory control over actions is to investigate individual differences. Evidence from psychopathology as well as non-clinical groups suggests that response inhibition is related to impulsivity. Clinical conditions that are characterized by dysfunctional impulsivity, such as attention deficit hyperactivity disorder (AD/HD; Nigg, 2005; Huizenga et al., 2009) and obsessive compulsive disorder (Penadés et al., 2006) show impaired stopping control compared to matched healthy groups. Even within a sample of healthy participants, Logan et al. (1997) reported that those people scoring higher on the impulsivity subscale from the extraversion scale of the Eysenck Personality Inventory (Eysenck and Eysenck, 1969) needed more time to inhibit their manual responses. Conversely, Go RT did not vary with impulsivity.

The fourth and last aim of the present report is to study verbal response inhibition in relation to individual differences in the personality trait of impulsivity. The current study extends earlier findings in two ways. First, we related individual differences in impulsivity to stopping proficiency over verbal responses, whereas previous studies are restricted to control over hand or eye movements. Second, we administered a version of Dickman's Impulsivity Inventory (Dickman, 1990) that dissociates functional and dysfunctional impulsivity. Dysfunctional impulsivity is defined as the tendency to act with less forethought than most people of equal 
ability when this tendency is a source of difficulty. In contrast, functional impulsivity relates to the tendency to act with relatively little forethought when such a style is optimal. The correlation between these two types of impulsivity is generally low (Dickman, 1990). Given the inhibitory difficulties in clinical groups that are characterized by dysfunctional impulsivity (Nigg, 2005; Penadés et al., 2006; Huizenga et al., 2009), we hypothesize that stopping proficiency correlates with scores on the dysfunctional subscale, rather than with the functional impulsivity subscale.

\section{EXPERIMENT 1 \\ MATERIAL AND METHODS \\ Participants}

Fourteen undergraduate students of the University of Amsterdam (mean age $=21.2$ years; $\mathrm{SD}=1.4$ years; 11 females, 3 males ) participated for course credit. All participants were native speakers of the Dutch language without language-related impairments. Written informed consent was obtained and this study was approved by the local ethics committee.

\section{Stimuli and procedure}

The binary manual and verbal stop tasks required respectively a manual or a verbal choice response (go responses) to pictures (go stimuli) and the inhibition of this response upon presentation of a stop-signal. Go stimuli were simple line drawings of a tree and a door $(7.06 \times 7.06 \mathrm{~cm})$ selected from a set described by Severens et al. (2005). Picture presentation was counterbalanced within a block of trials and presented pseudo-randomly against a lightgray background. Go stimulus presentation terminated after $1 \mathrm{~s}$ or directly upon a response. Responses were recorded during an interval of $1400 \mathrm{~ms}$ following picture onset. The inter-trial interval varied randomly between 1750 and $1250 \mathrm{~ms}$.

All participants completed the manual and the verbal versions of the stop task. Two button boxes recorded left and right index finger responses in the manual version. Stimulus-response mapping was counterbalanced across subjects. In the verbal version, participants responded by naming each picture out loud, saying "boom" (Dutch for tree) or "deur" (Dutch for door). Naming latencies were measured using a voice key. Speech was recorded for $1400 \mathrm{~ms}$ after picture onset to monitor responses. Responses were coded offline as incorrect if a wrong picture name was produced. If the voice key was triggered incorrectly due to hesitation, stuttering or non-verbal responses, the trial was excluded from analysis (3.7\%).

A visual stop-signal was presented on $30 \%$ of the trials, upon which participants tried to inhibit the go response. The stop-signal consisted of a red square frame $(0.85 \mathrm{~cm})$ surrounding the picture border. The interval between the onset of the go picture and the onset of the stop-signal (i.e., stop-signal delay) was set at $200 \mathrm{~ms}$ on the first stop trial. From then on, an online tracking algorithm adjusted stop-signal delay as a function of individual stopping performance (Levitt, 1971). After successful inhibition, stop-signal delay increased by $50 \mathrm{~ms}$, thereby decreasing the chances of successful inhibition on the next stop trial. Stop-signal delay decreased by $50 \mathrm{~ms}$ if the participant was unable to stop (i.e., failed-inhibition trial), increasing the chances of stopping. This adaptive algorithm ensured successful inhibition on about $50 \%$ of the stop trials, a procedure that yields reliable estimates of SSRT (Band et al., 2003).
Given that the response could be withheld on $n \%$ of all stop trials, SSRT is calculated by subtracting mean stop-signal delay from the Go RT marking the $n$-th percentile (see Figure 1 for an illustration of the integration method).

A block of trials consisted of 60 trials, including 42 go and 18 stop trials. The manual and verbal stop tasks each included one practice block and three test blocks. Task order was counterbalanced across participants. Prior to practice, participants were familiarized with the pictures. Participants completed the self-report impulsivity questionnaire (Dickman, 1990) that measures functional and dysfunctional impulsivity. Dysfunctional impulsivity is defined as the tendency to act with little forethought when such a tendency is disadvantageous. Functional impulsivity, in contrast, is the tendency to act with relatively little forethought when such a style is optimal. Total experiment duration was $1 \mathrm{~h}$.

\section{RESULTS}

Mean RT on go trials (i.e., trials without a stop-signal), square-rooted error rates, SSRT, and mean RT on failed-inhibition trials were analyzed using repeated-measures ANOVA with Response Modality (manual vs. verbal) as within-subject factor (see Table 1).

Overall, verbal responses were $64 \mathrm{~ms}$ slower than manual responses, $F(1,13)=19.26, p<0.001$. Verbal responses were also less prone to choice errors $(1.2 \%)$ than manual responses $(4.6 \%)$, $F(1,13)=16.05, p<0.001$. This pattern of results may indicate a trade-off between speed and accuracy. Verbal and manual Go RT were highly correlated $(r=0.67, p<0.01)$ indicating that participants who were fast at pressing buttons were also relatively fast in naming the pictures out loud.

Participants successfully inhibited their overt responses on about half of the stop trials, indicating that the tracking algorithm worked well for both response modalities (manual 49\% vs. verbal 51\%, $F(1,13)=1.26, p=0.28$. In line with the predictions of the race model, RT on trials that escaped inhibition (i.e., failed-inhibition RT) was shorter than RT on go trials, $F(1,13)=24.48, p<0.001$. This is because failed-inhibition RTs are taken from trials on which participants were unable to stop, derived from the fast (left) part of

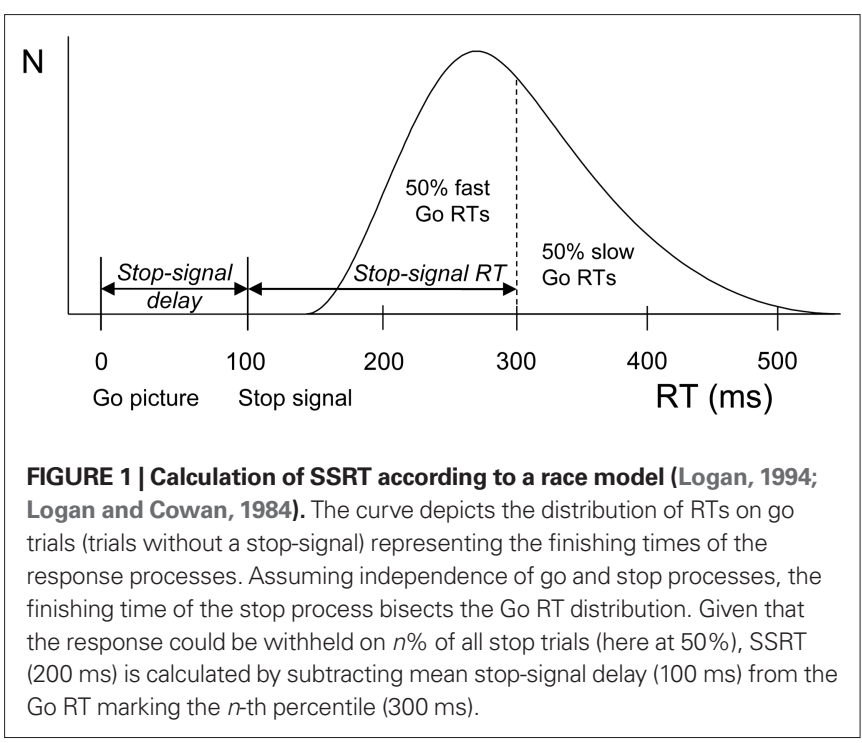


the RT distribution of go trials (see Figure 1). Manual and verbal SSRTs were very similar, about $194 \mathrm{~ms}(F<1)$ but did not correlate significantly across participants (see Figure $2 \mathrm{~A}, r=0.06, p=0.85$ ).

Table 1 | Mean Go RT and Error Percentages on Go trials, Percentage of Stop-Inhibit Trials, Stop-Signal Delay, Failed-Inhibition RT, and StopSignal RT in the Binary Manual and Verbal Stop Tasks (Experiment 1) and in the High-Frequency (HF) and Low-Frequency (LF) Conditions of the Picture-Naming Stop Task (Experiment 2). SEM in parentheses.

\begin{tabular}{llllll}
\hline & \multicolumn{2}{c}{$\begin{array}{c}\text { Experiment 1 } \\
\text { (binary stop task) }\end{array}$} & & \multicolumn{2}{c}{$\begin{array}{c}\text { Experiment 2 } \\
\text { (picture-naming } \\
\text { stop task) }\end{array}$} \\
\cline { 2 - 3 } & Manual & Verbal & & HF & LF \\
\hline Go RT & $452(18)$ & $516(18)$ & & $613(15)$ & $620(14)$ \\
Go errors (in \%) & $4.6(0.8)$ & $1.8(0.7)$ & & $1.7(0.3)$ & $0.9(0.3)$ \\
Stop-inhibit (in \%) & $49(1)$ & $51(1)$ & & $54(6)$ & $53(7)$ \\
Stop-signal delay & $242(22)$ & $292(20)$ & & $330(17)$ & $326(16)$ \\
Failed-inhibition RT & $400(13)$ & $482(24)$ & & $550(12)$ & $563(11)$ \\
Stop-signal RT & $193(10)$ & $194(12)$ & & $236(14)$ & $253(12)$
\end{tabular}

When comparing response and stopping latencies obtained in the two tasks with scores on the impulsivity questionnaire, only one significant correlation was obtained between verbal SSRT and dysfunctional impulsivity (see Figure 2B, $r=0.61, p=0.02$ ). Participants who had a higher score on the dysfunctional impulsivity scale were less proficient at inhibiting their vocal responses.

\section{DISCUSSION}

Experiment 1 compared stopping control over binary manual and verbal responses. Participants responded to two pictures by pressing a left or right button (manual task) or by naming the stimulus representation out loud (verbal task). We observed an average verbal choice RT (516 ms) that was about $64 \mathrm{~ms}$ slower than the average button-press response. Further, we observed comparable stopping latencies for manual and verbal binary responses of 193 and $194 \mathrm{~ms}$ respectively, which are very close to values reported in numerous previous stop studies (see also Logan, 1994).

The results obtained in Experiment 1 differ in two ways from the pattern reported by Xue et al. (2008) who obtained similar manual and verbal response latencies to letters (both about $400 \mathrm{~ms}$ ). As far as the stopping data are concerned, they reported shorter stopping latencies for naming a letter compared to stopping a manual
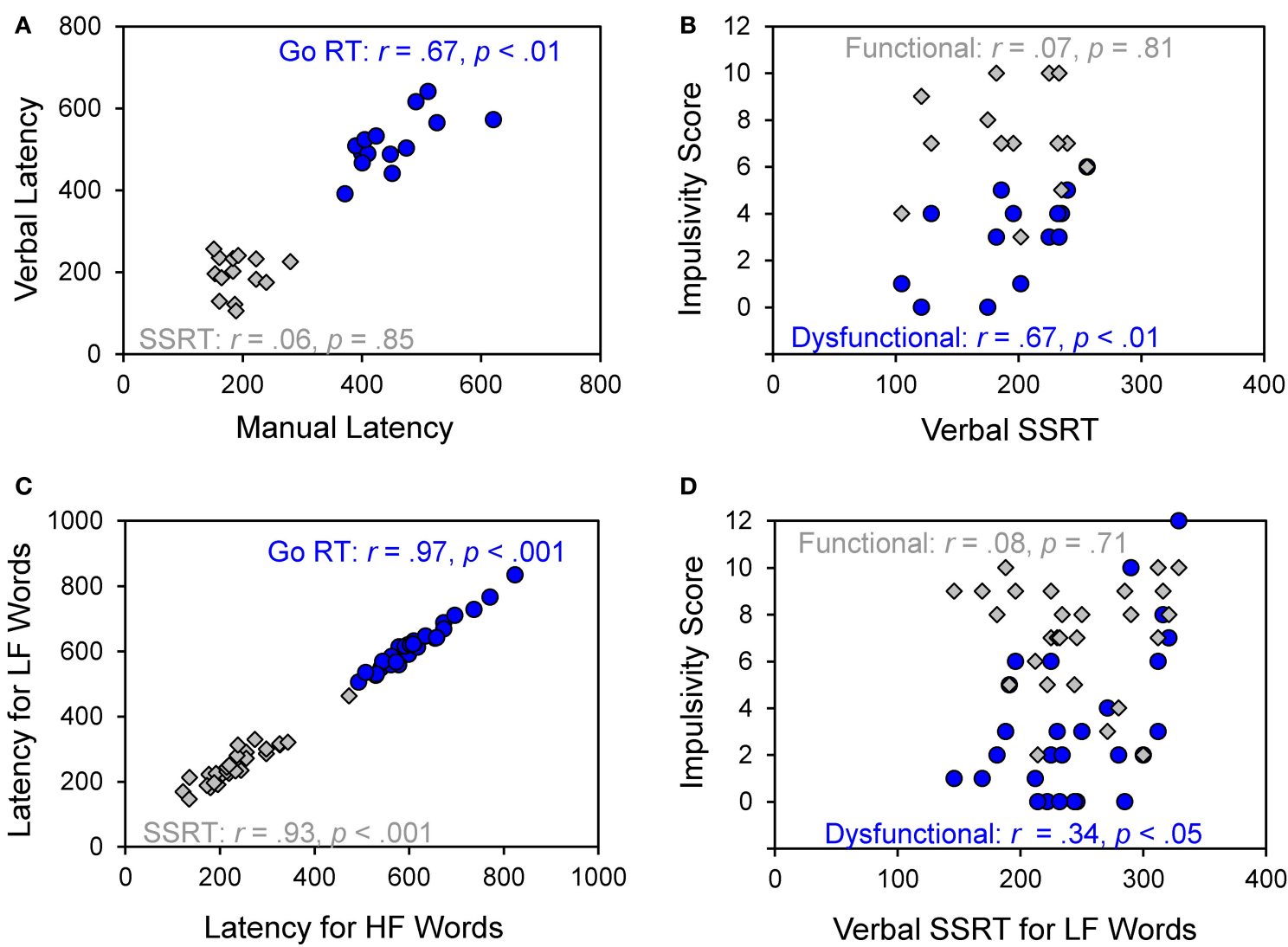

D

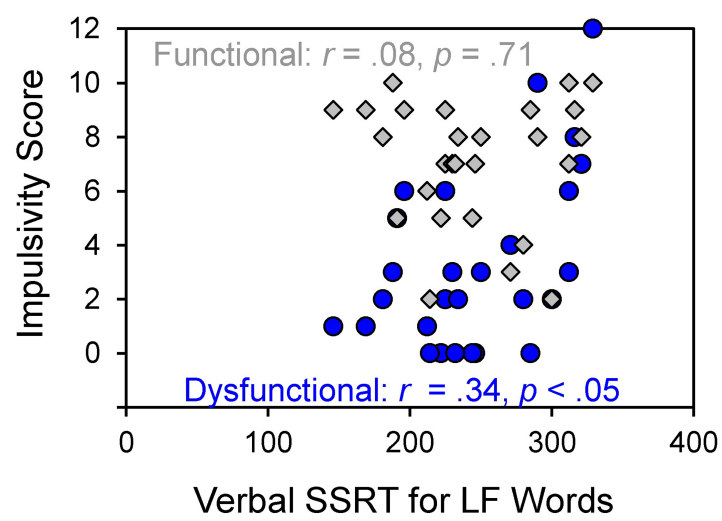

FIGURE 2 | (A) Correlations between manual and verbal Go RT (blue circles) and SSRT (gray diamonds) for choice responses in Experiment 1. (B) Correlations between verbal SSRT and impulsivity scores (functional scores in gray diamonds; dysfunctional scores in blue circles) in Experiment 1. (C) Correlations for Go RT (blue circles) and SSRT (gray diamonds)

between high-frequency (HF) and low-frequency (LF) conditions in Experiment 2. (D) Correlations between verbal SSRT for low-frequency (LF) words and impulsivity scores (functional impulsivity scores in gray diamonds; dysfunctional impulsivity scores in blue circles) in Experiment 2. 

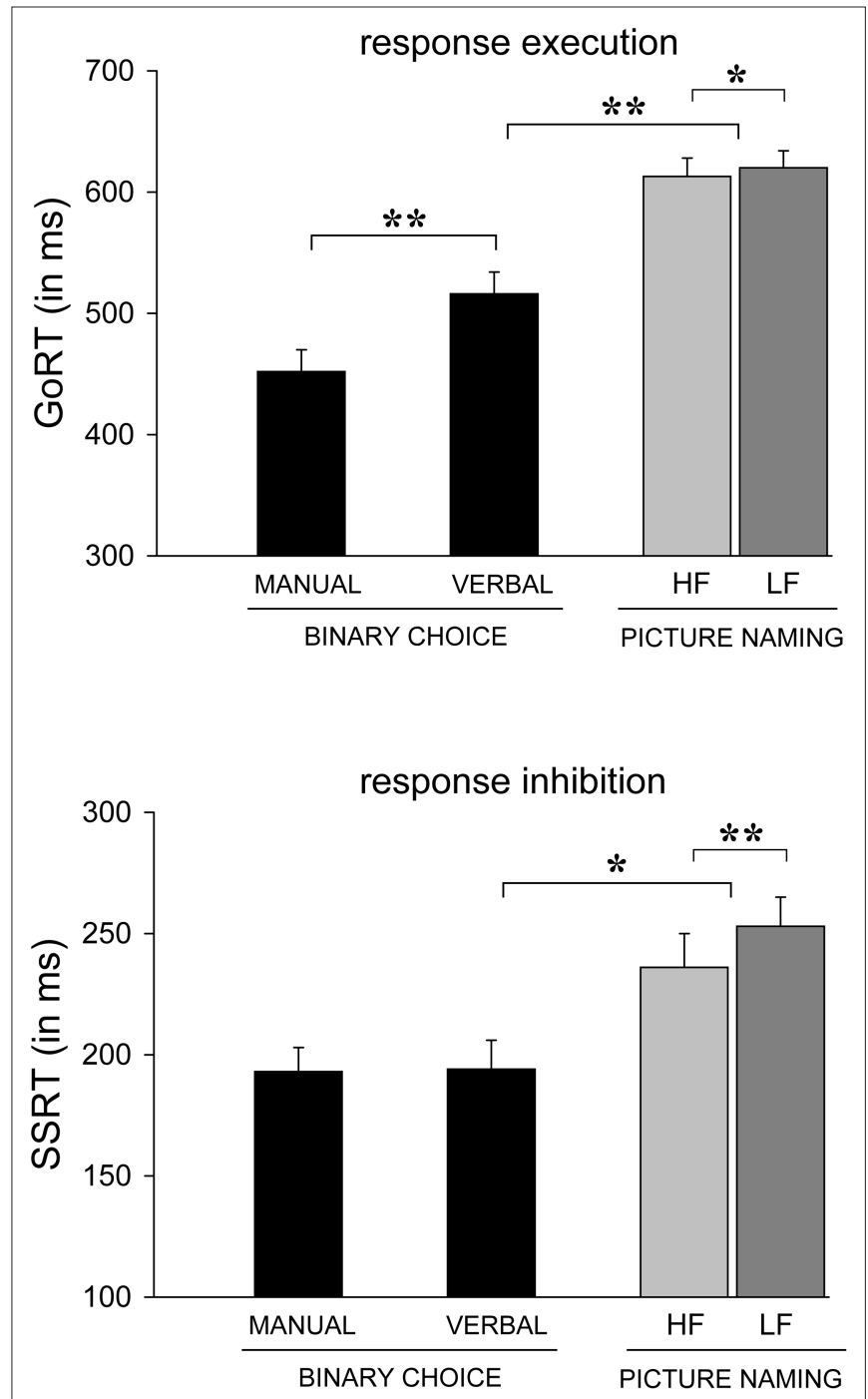

FIGURE 3 | Mean Go RT (upper panel) and SSRT (lower panel) for manual and verbal binary responses in the binary-choice tasks (Experiment 1) and for verbal responses to high-frequent (HF, light-gray bars) and low-frequent (LF, dark-gray bars) pictures in the picture-naming task (Experiment 2). ${ }^{*} p<0.05 ;{ }^{*} p<0.01$.

response but did not elaborate on the relatively shorter SSRT for letter naming versus responding manually. Instead, we observed comparable stopping latencies for verbal and manual binary responses. This discrepancy between studies might be related to differences between go tasks. First, a length effect on word production may account for the longer response latencies for producing whole picture words compared to naming single letters (e.g., Meyer et al., 2003). Another difference between picture naming and letter naming is that our visual stimuli of a tree and a door were more complex (i.e., had more lines and more detail) than the single letter stimuli used by Xue et al. (2008), which may have yielded an effect of visual complexity on naming latency (Attneave, 1957). Thus word length and stimulus complexity might have contributed to our observation of longer verbal RTs compared to the relatively shorter letter naming RTs reported by Xue et al. (2008).
Experiment 2 included multiple go pictures to be named. In addition, the linguistic complexity of the go pictures names was manipulated by using pictures that represented high and lowfrequency object names.

\section{EXPERIMENT 2 \\ MATERIALS AND METHODS \\ Participants}

A different sample of 28 students (mean age $=22.1$ years, $\mathrm{SD}=4.6$ years; 20 females, 8 males) participated for course credit. Inclusion criteria and informed consent procedures were similar to Experiment 1.

\section{Stimuli and procedure}

Sixty pictures consisting of simple white-on-black line drawings were selected from the stimulus database described by Severens et al. (2005). The set represented 30 high-frequent (HF) and 30 low-frequent (LF) monosyllabic and bisyllabic object names (see Table 2) that were randomized within a trial block. Mean occurrences per million for HF and LF pictures were respectively 228 and 13 (Baayen et al., 1995). LF and HF picture sets were matched in terms of name agreement (respectively $97.2 \%$ and 98.5\%) and length (i.e., number of phonemes, 3.5 for both HF and LF). Similar to Experiment 1, a voice key recorded verbal responses. A visual stop-signal was presented on $30 \%$ of the trials, upon which participants tried to inhibit their verbal response. Stop-signal delay was controlled online by two independent tracking algorithms that worked separately for HF and LF picture categories.

Before testing, the pictures were shown to the participants together with their correct names to prevent naming ambiguity. A block of trials contained 42 go trials ( $21 \mathrm{HF}$ vs. $21 \mathrm{LF}$ ) and 18 stop trials ( $9 \mathrm{HF}$ vs. $9 \mathrm{LF}$ ). The picture-naming stop task included one practice block and six test blocks of 60 trials each in order to obtain sufficient stop trials for a reliable computation of SSRTs for the HF and LF conditions (Band et al., 2003). Questionnaire and task administration, including short breaks between blocks, lasted about $70 \mathrm{~min}$.

\section{RESULTS}

Mean verbal RT, square-rooted error percentages to go signals as well as SSRTs and mean failed-inhibition RTs were analyzed using a repeated-measures ANOVA with Frequency (HF vs. LF) as withinsubject factor (Figure 3).

A small but reliable Frequency effect indicated that LF pictures were named 7-ms slower than HF pictures (respectively, 620 vs. $613 \mathrm{~ms}, F(1,27)=6.52, p=0.02)$. Analyses of error rates (less than $2 \%$ ) revealed more naming errors in the LF than in the HF condition, $F(1,27)=5.46, p=0.03$. This pattern argues against an interpretation in terms of a speed-accuracy trade-off. A follow-up analysis was performed to test if the small overall frequency effect on naming RT is related to the repetition of pictures in Experiment 2. Repeated-measures ANOVA of the frequency effect as a function of test block (1-6) did not confirm a reduction of the frequency effect on naming with repetition, as indicated by the non-significant interaction, $F(5,135)=1.11$, $p=0.36$. Thus, the frequency effect on naming latency was stable over time. 
Table 2 | Names of the pictures presented as go stimuli in Experiment 2 (taken from Severens et al., 2005).

\begin{tabular}{|c|c|c|c|}
\hline \multicolumn{2}{|c|}{ High-frequency (HF) pictures } & \multicolumn{2}{|c|}{ Low-frequency (LF) pictures } \\
\hline Dutch & English & Dutch & English \\
\hline auto & car & aap & ape \\
\hline bank & bench & cactus & desert \\
\hline bed & bed & clown & clown \\
\hline blad & leaf & draak & dragon \\
\hline bloem & flower & gitaar & guitar \\
\hline boek & book & haak & hook \\
\hline boom* & tree & helm & helmet \\
\hline deur* & door & iglo & igloo \\
\hline fles & bottle & kaars & candle \\
\hline glas & glass & kam & comb \\
\hline hand & hand & kip & chicken \\
\hline hart & heart & kok & cook \\
\hline hond & dog & kroon & crown \\
\hline huis & house & leeuw & lion \\
\hline kerk & church & pauw & peacock \\
\hline koning & king & peer & pear \\
\hline muur & wall & pet & hat \\
\hline neus & nose & pijl & arrow \\
\hline oog & eye & pijp & pipe \\
\hline oor & ear & robot & robot \\
\hline paard & horse & sjaal & scarf \\
\hline stoel & chair & slak & snail \\
\hline tand & tooth & slee & sled \\
\hline tafel & table & tent & tent \\
\hline trap & stairs & uil & owl \\
\hline trein & train & vork & fork \\
\hline vis & fish & vos & fox \\
\hline voet & foot & wiel & wheel \\
\hline vuur & fire & zaag & saw \\
\hline zak & bag & zwaan & swan \\
\hline
\end{tabular}

Pictures used as go stimuli in Experiment 1 are indicated with *

The tracking algorithms in the HF and the LF conditions converged to inhibition rates of respectively $54 \%$ and $53 \%, F<1$. Overall, failed-inhibition RT (557 ms) was shorter than Go RT (617 ms), which meets the predictions of the race model. $F(1,27)=54.35$, $p<0.001$. Analysis of SSRT indicated that stopping the naming of LF pictures was $17 \mathrm{~ms}$ slower than stopping HF picture naming (respectively $253 \mathrm{~ms}$ vs. $236 \mathrm{~ms}, F(1,27)=11.61, p=0.002)$. A subsequent analysis of covariance (ANCOVA) was performed to test whether these frequency effects on stopping latency are related to the observed frequency effects on naming latency. ANCOVA of SSRT, with naming RT entered as a covariate, still yielded a significant effect of word frequency on stopping; SSRT for LF words was significantly prolonged compared to SSRT for HF words, $F$ (1, 27) $=10.21, p=0.004$. This asserts that the frequency effect on stopping cannot be explained in terms of the frequency effect on naming RT and that the frequency effect on stopping and naming are independent (see van den Wildenberg et al., 2006 for a similar statistical approach).
Figure 2C shows that word production latencies of $\mathrm{HF}$ and $\mathrm{LF}$ words are highly correlated $(r=0.97, p<0.001)$ and so are HF and LF stopping latencies $(r=0.93, p<0.001)$. Naming RT did not correlate significantly with SSRT ( $p s>0.10$ ). Similar to Experiment 1 , scores on the dysfunctional impulsivity subscale correlated with verbal SSRT (see Figure 2D), indicating that participants with higher scores showed prolonged SSRT in the LF naming condition $(r=0.34, p=0.04)$.

\section{DISCUSSION}

Repeated-measures ANOVA of the frequency effect as a function of test block (1-6) did not confirm a reduction of the frequency effect on naming with repetition, as indicated by the non-significant interaction, $F(5,135)=1.11, p=0.36$. Thus, the frequency effect on naming latency was stable over time.

Experiment 2 replicates a typical finding in the picture-naming literature, namely that it generally takes more time to produce object words with low-frequency names compared to pictures representing words of higher frequency (e.g., Oldfield and Wingfield, 1965; Jescheniak and Levelt, 1994). The frequency effect on naming latency has been primarily attributed to the lexical selection stage, although the exact locus has been a matter of debate (e.g., Jescheniak and Levelt, 1994; Barry et al., 1997; Griffin and Bock, 1998; Caramazza et al., 2001; Navarrete et al., 2006; Graves et al., 2007; Dent et al., 2008). Although the obtained frequency effect on naming of $7 \mathrm{~ms}$ was statistically significant, it should be noted that this difference is relatively small. We tested whether this small effect size might be related to the repetition of pictures in Experiment 2. For example, Griffin and Bock (1998) observed that repetition substantially reduced the frequency effect on picture-naming latency. However, follow-up analysis of the frequency effect as a function of block indicated that the frequency effect on word production was stable over time (see also Jescheniak and Levelt, 1994). The frequency effect on picture naming that was obtained in the current study is relatively smaller in magnitude than commonly reported in the picture-naming literature. This reduction may be related to the insertion of stop-signals in the task. For example, Logan and Irwin (2000) and van den Wildenberg and van der Molen (2004) also observed that effects of experimental factors that otherwise yield sizeable effects on Go RT (like the instruction to respond in a compatible or incompatible fashion to visual stimuli) were strongly reduced when stop-signals were intermixed in the task. The effects of word frequency on verbal stopping will be discussed in the following section.

\section{GENERAL DISCUSSION}

This study centered on the ability to exert stopping control over speech by combining two well-established research paradigms; the stop-signal task that taps the ability to inhibit ongoing behavior (Logan and Cowan, 1984), and the picture-naming task that is widely used to examine word production (Glaser, 1992). Our goals were (1) to compare inhibitory control over manual and verbal responses, (2) to investigate whether verbal stopping is affected by the number of response alternatives, (3) to identify linguistic processing factors that affect inhibitory control over speech, and (4) to assess the relation between individual differences in impulsivity and verbal stopping proficiency over speech. 
In a two-choice setup (Experiment 1), subjects were relatively faster generating a manual response compared to producing a verbal response to a picture. We obtained reliable manual and verbal SSRTs to stop-signals that were very close to $200 \mathrm{~ms}$ for both manual and verbal binary choice responses. This observation is in line with a large body of stop literature indicating that a variety of binary overt actions can be countermanded in about 200 ms (see Logan, 1994 for a review).

Experiment 2 extended the stimulus set to multiple pictures to be named, thereby increasing the ecological validity of the task compared to a binary go instruction. As predicted, increasing the number of different pictures to be named (Experiment 2) prolonged naming latency. A likely explanation for this increase in verbal naming latency is that the associations between two picture stimuli (e.g., of a door and a tree) and two verbal responses (e.g., saying "door" vs. "tree") can be actively maintained by and rapidly retrieved from working memory. Fast retrieval of the appropriate mapping rule from working memory yields relatively fast object discrimination and naming. Conversely, naming one out of multiple pictures, the stimulus-response mapping rules cannot be stored in working memory but instead have to be retrieved from the lexicon upon presentation of a picture. This additional processing time is reflected in longer picture-naming latencies for a large picture set (Experiment 2) compared to a binary naming instruction (Experiment 1). Interestingly, increasing the picture repertoire from two to sixty prolonged stopping latency of speech by about $50 \mathrm{~ms}$. Clearly, the extra demands related to lexical retrieval of the correct picture name prolonged inhibition latencies compared to a verbal two-choice situation. The observation that SSRT increases as the number of alternative responses increases is in line with a study by Logan et al. (1984). They reported slower stopping for choice responses that are issued by two fingers compared to stopping in a simple task variant in which participants always responded with one finger (see also Szmalec et al., 2009).

The third aim of this study was to assess the effect of linguistic processing demands on stopping control over speech by manipulating relative word frequency. As has been repeatedly reported, participants were slightly but significantly slower when naming LF object words in the go task compared to naming HF words. This frequency effect on word production has been explained in terms of increased lexical processing demands for producing LF words (Navarrete et al., 2006; Almeida et al., 2007; Graves et al., 2007). Of particular interest is the effect of word frequency on inhibitory control over speech; stopping was significantly slowed for producing LF words compared to naming HF pictures. This observation presents a case of functional dependence between going and stopping. Functional dependence refers to a situation in which a single experimental factor affects the latencies of both the go process and the stop process. For example, Ridderinkhof et al. (1999) reported that Go RT as well as SSRT were longer for incongruent trials compared to congruent trials in an Eriksen interference task. This result suggests that the suppression of an interfering response in the go task and response inhibition upon a stop-signal share a common set of limited inhibitory resources (Ridderinkhof et al., 1999).

It should be noted that functional dependence between going and stopping does not necessarily violate the independence assumptions of the race model (Logan and Cowan, 1984; Logan,
1994; Verbruggen and Logan, 2009). The model's independence assumptions for computing SSRT are two-fold and refer to (1) context independence, assuming that the distribution of Go RTs is the same for trials with and for trials without a stop-signal, and (2) stochastic independence, assuming that trial-by-trial variability in Go RT is unrelated to trial-by-trial variability in SSRT. The present data are in line with the predictions of these two independence assumptions: (a) in both experiments we observed that mean Go RT was longer than RT on failed-inhibition trials, and (b) the covariate analysis reported in Experiment 2 indicates that the prolonged stopping observed for naming low-frequency words cannot be explained by the prolonged naming latencies in this condition. These two results indicate that our data do not violate the independence assumptions of the race model and that our estimates of SSRT appear to be reliable.

The notion of functional dependence is in line with current theories on speech self-monitoring and recent findings in speech production. Hartsuiker and Kolk (2001) report a computational model of speech monitoring together with explicit assumptions about the coordination of stopping ongoing speech and speech repair. More specifically, they argue that stopping, proceeding, and repairing speech processes proceed in parallel. This notion is very compatible with the assumptions of the race model (Logan and Cowan, 1984; Logan, 1994; Boucher et al., 2007). Furthermore, Hartsuiker et al. (2008b) showed that if one process becomes more difficult (e.g., proceeding) then the stopping process is prolonged, suggesting that stopping, proceeding, and repairing speech processes share a common set of resources, in other words, are functionally dependent (Hartsuiker et al., 2005, 2008a). The current finding that stopping is slower if the target word is of lowfrequency seems to provide additional evidence for that theory, given that lexical frequency is proposed to load on central resources (Ferreira and Pashler, 2002; Dent et al., 2008). The frequency effect on verbal SSRT also relates to recent studies indicating that verbal monitoring is a special case of general performance monitoring which implicates brain areas associated with general cognitive control (Christoffels et al., 2007; Ganushchak and Schiller, 2008; Riès et al., in press).

A interesting study in this respect is provided by Slevc and Ferreira (2006) who used the stop-signal paradigm to test predictions derived from the perceptual loop theory of speech monitoring (Levelt, 1983). They combined a picture-naming task with the presentation of auditory or visual stop-signal words that differed from the picture name. Interestingly, stopping proficiency depended on the level of phonological similarity between the name of the go picture and stop-signal word; it was harder to stop if the stop-signal was phonologically similar to the to-be-named word than following a stop-signal that was phonologically dissimilar. In contrast, overlap in terms of semantic similarity did not affect stopping latency. According to the authors, this pattern of similarity-based vulnerability suggests that the perceptual loop detects errors by making comparisons at the phonological level (Slevc and Ferreira, 2006). Albeit interesting from a speech monitoring point of view, their stopping data are somewhat difficult to interpret in terms of inhibitory control over speech. During picture naming, speakers were presented with valid and invalid stop-signal words and should stop only if the presented stop word was different from the picture name. 
Inhibitory processing thus included the additional processing stage of stop-signal discrimination which requires additional processing time (e.g., van de Laar et al., 2010).

Finally, the present study provides direct evidence that inhibitory action control over speech production varies specifically with dysfunctional impulsivity as a personality trait. Verbal stopping latencies were longer for subjects that scored higher on a self-report questionnaire that measured dysfunctional impulsivity (see also Logan et al., 1997). Thus, speech is harder to control for those individuals who tend to base their reactions on rapid information processing and initial impressions rather than on a slower, more adaptive approach (Dickman, 1990). In contrast, functional impulsivity scores, reflecting the tendency to respond quickly (albeit inaccurately) in situations where this is optimal, were not correlated with stopping latencies. The observation that individual differences in dysfunctional impulsivity predict stopping control over speech extends previous studies that are restricted to hand movements (e.g., Logan et al., 1997). It also shows that specifically dysfunctional impulsivity, and not functional impulsivity, is associated with prolonged stopping latencies, even in a non-clinical sample. This conclusion is in line with multiple studies that report deficient stop-signal inhibition in various clinical groups that are associated with impulsivity problems.

A limitation of the present study is that we did not control our picture material for age of acquisition, a factor that correlates with word frequency but has shown to be dissociable (e.g., Dent et al., 2008). We did not do so because this would pose high constraints on the selection of picture stimuli, which would interfere with our

\section{REFERENCES}

Alario, F.-X., Ferrand, L., Laganaro, M., New, B., Frauenfelder, U.H., and Segui, J. (2004). Predictors of picture naming speed. Behav. Res. Methods Instrum. Comput. 36, 140-155.

Almeida, J., Knobel, M., Finkbeiner, M., and Caramazza, A. (2007). The locus of the frequency effect in picture naming: When recognizing is not enough. Psychon. Bull. Rev. 14, 1177-1182.

Attneave, F. (1957). Physical determinants of the judged complexity of shapes. J. Exp. Psychol. 53, 221-227.

Baayen,R.H.,Piepenbrock, R., andGulikers, L. (1995). The Celex Lexical Database (Release2) [CD-ROM]. Linguistic Data Consortium. Philadelphia: University of Pennsylvania.

Band, G. P. H., van der Molen, M. W., and Logan, G. D. (2003). Horse-race model simulations of the stop-signal procedure. Acta Psychol. 112, 105-142.

Barry, C., Morrison, C. M., and Ellis, A. W. (1997). Naming the Snodgrass and Vanderwart pictures: effects of age of acquisition, frequency, and name agreement. Q. J. Exp. Psychol. 50, 560-585.

Boucher, L., Palmeri, T. J, Logan, G. D., and Schall, J. D. (2007). Inhibitory control in mind and brain: An interactive race model of countermanding saccades. Psychol. Rev. 114, 376-397.
Caramazza, A., Costa, A., Miozzo, M., and Bi, Y. (2001). The specific word frequency effect: implications for the representation of homophones in speech production. J. Exp. Psychol. Learn Mem. Cogn. 27, 1430-1450.

Christoffels, I. K., Formisano, E., and Schiller, N. O. (2007). The neural correlates of verbal feedback processing: an fMRI study employing overt speech. Hum. Brain Mapp. 28, 868-879.

Dent, K., Johnston, R.A., and Humphreys, G. W. (2008). Age of acquisition and word frequency effects in picture naming: A dual-task investigation. J. Exp. Psychol. Learn Mem. Cogn. 34, 282-301.

Dickman, S. J. (1990). Functional and dysfunctional impulsivity: Personality and cognitive correlates. J. Pers. Soc. Psychol. 58, 95-102.

Eysenck, H. J., and Eysenck, S. B. (1969). Personality Structure and Measurement. San Diego: Knapp.

Ferreira, V. S., and Pashler, H. (2002). Central bottleneck influences on the processing stages of word production. J. Exp. Psychol. Learn Mem. Cogn. 28, 1187-1199.

Ganushchak, L. Y., and Schiller, N. O. (2008). Motivation and semantic context affect brain error-monitoring

goal to obtain a large set of high-quality items. We therefore cannot exclude that our frequency effects may also be related to differences in age of acquisition. In addition, control over word production that is cued externally, as in picture naming, might differ from controlling speech acts that are generated internally.

In closing, the present study illustrates a fruitful combination of two well-established research paradigms (i.e., the stop-signal task and the picture-naming task) to study variables that modulate inhibitory control over speech production. Note that although picture naming offers a large set of natural stimulus-response combinations, it is limited to the production of single words. It would be interesting to extent these findings to inhibiting the production of more complex speech like phrases or whole sentences. Future studies may adopt this approach, either to further examine how inhibitory control over speech relates to factors that are known to affect word production, such as concept familiarity and age of acquisition, or to examine group or individual differences in stopping proficiency over speech.

\section{ACKNOWLEDGMENTS}

This study was supported by VENI grants from the Dutch Organization for Scientific Research (NWO) to the first and second authors. We thank Bert van Beek for programming the tasks and Janneke Gulen, Ilonka de Haas, Ceciel Harmsen, Janneke Heerkens, and Phineke Tielenius Kruythoff for their enthusiasm and invaluable assistance in recruiting and testing the participants of this study. Els Severens is gratefully acknowledged for granting access to her picture data set.

activity: an event-related brain potentials study. Neuroimage 39, 395-405.

Glaser, W. R. (1992). Picture naming. Cognition 42, 61-105.

Graves, W. W., Grabowski, T. J., Mehta, S., and Gordon, J. K. (2007). A neural signature of phonological access: Distinguishing the effects of word frequency from familiarity and length in overt picture naming. J. Cogn Neurosci. 19, 617-631.

Griffin, Z. M., and Bock, K. (1998). Constraint, word frequency, and the relationship between lexical processing levels in spoken word production. J. Mem. Lang. 38, 313-338.

Hartsuiker, R. J., Catchpole, C. M., de Jong, N. H., and Pickering, M. J. (2008a). Concurrent processing of words and their replacements during speech. Cognition 108, 602-607.

Hartsuiker, R. J., Pickering, M. J., and De Jong, N. H. (2005). Semantic and phonological context effects in speech error repair. J. Exp. Psychol. Learn. Mem. Cogn. 31, 921-932.

Hartsuiker, R. J., Pickering, M. J., and de Jong, N. H. (2008b). Semantic and phonological context effects in speech error repair. J. Exp. Psychol. Learn Mem. Cogn. 31, 921-932.

Hartsuiker, R. J., and Kolk, H. H. J. (2001). Error monitoring in speech production: a computational test of the perceptual loop theory. Cogn. Psychol. 42, 113-157.

Huizenga, H. M., van Bers, B. C. W., Plat, J., van den Wildenberg, W. P. M., and van der Molen, M. W. (2009). Task complexity enhances response inhibition deficits in attention deficit hyperactivity disorder (ADHD): A meta regression analysis. Biol. Psychiatry 65, 39-45.

Indefrey, P., and Levelt, W. J. M. (2004). The spatial and temporal signatures of word production components. Cognition 92, 101-104.

Jescheniak, J. D., and Levelt, W. J. M. (1994). Word frequency effects in speech production: retrieval of syntactic information and of phonological form. J. Exp. Psychol. Learn Mem. Cogn. 20, 824-843.

Ladefoged, P., Silverstein, R., and Papçun, G. (1973). Interruptibility of speech. J. Acoust. Soc. Am. 54, 1105-1108.

Levelt, W. J. M. (1983). Monitoring and self-repair in speech. Cognition 14, 41-104.

Levelt, W. J. M. (1992). Accessing words in speech production: Stages, processes and representations. Cognition 42, 1-22.

Levelt, W. J. M. (1999). Models of word production. Trends Cogn. Sci. 3, 223-232. 
Levitt, H. (1971). Transformed up-down methods in psychoacoustics. J. Acoust. Soc. Am. 49, 467-477.

Logan, G. D. (1994). "On the ability to inhibit thought and action: a users' guide to the stop signal paradigm," in Inhibitory Processes in Attention, Memory and Language, eds D. Dagenbach and T.H. Carr (San Diego, CA: Academic Press), 189-239.

Logan, G. D., and Cowan, W. B. (1984). On the ability to inhibit thought and action: a theory of an act of control. Psychol. Rev. 91, 295-327.

Logan, G. D., Cowan, W. B., and Davis, K. A. (1984). On the ability to inhibit simple and choice reaction time responses: a model and a method. J. Exp. Psychol. Hum. Percept. Perform. 10, 276-291.

Logan, G. D., and Irwin, D. E. (2000). Don't look! Don't touch! Inhibitory control of eye and hand movements. Psychon. Bull. Rev. 7, 107-112.

Logan, G. D., Schachar, R. J., and Tannock, R. (1997). Impulsivity and inhibitory control. Psychol. Sci. 8, 60-64.

Meyer, A. S., Roelofs, A., and Levelt, W. J. M. (2003). Word length effects in object naming: the role of a response criterion. J. Mem. Lang. 48, 113-147.

Navarrete, E., Basagni, B., Alario, F. X., and Costa, A. (2006). Does word frequency affect lexical selection in speech production? Q. J. Exp. Psychol. 59, 1681-1690.
Nigg, J. T. (2005). Neuropsychologic theory and findings in attention deficit/hyperactivity disorder: The state of the field and salient challenges for the coming decade. Biol. Psychiatry 57, $1424-1435$

Oldfield, R. C., and Wingfield, A. (1965). Response latencies in naming objects. Q. J. Exp. Psychol. 17, 273-281.

Penadés, R., Catalán, R., Rubia, K., Andrés, S., Salamero, M., and Gastó, C. (2006). Impaired response inhibition in obsessive compulsive disorder. Eur. Psychiatry 22, 404-410.

Ridderinkhof, K. R., Band, G. P. H., and Logan, G. D. (1999). A study of adaptive behavior: effects of age and irrelevant information on the ability to inhibit one's actions. Acta Psychol. 101, 315-337.

Riès, S., Janssen, N., Dufau, S., Alario, F. X., and Burle, B. (in press). Generalpurpose monitoring during speech production. J. Cogn. Neurosci. doi: 10.1162/jocn.2010.21467. [Epub ahead of print].

Severens, E., van Lommel, S., Ratinckx, E., and Hartsuiker, R. J. (2005). Timed picture naming norms for 590 pictures in Dutch. Acta Psychol. 119, 159-187.

Slevc, L. R., and Ferreira, V. S. (2006). Halting in single word production: a test of the perceptual loop theory of speech monitoring. J. Mem. Lang. 54, 515-540.
Szmalec, A., Demanet, J., Vandierendonck, A., and Verbruggen, F. (2009). Investigating the role of conflict resolution in memory updating by means of the one-back choice RT task. Psychol. Res. 73, 390-406.

van de Laar, M. C., van den Wildenberg, W. P. M., van Boxtel, G. J. M., and van der Molen, M. W. (2010). Processing of global and selective stop signals: application of Donders' subtraction method to stop-signal task performance. Exp. Psychol. 57, 149-159.

van den Wildenberg, W. P. M., van Boxtel, G. J. M., van der Molen, M. W., Bosch, D. A., Speelman, J. D., and Brunia, C. H. M. (2006). Stimulation of the subthalamic region facilitates the selection and inhibition of motor responses in Parkinson's disease. J. Cogn. Neurosci. 18, 626-636.

van den Wildenberg, W.P.M., and van der Molen, M. W. (2004). Developmental trends in simple and selective inhibition of compatible and incompatible responses. J. Exp. Child. Psychol. 87, 201-220.

Verbruggen, F., and Logan, G. D. (2008). Response inhibition in the stopsignal paradigm. Trends Cogn. Sci. 12, 418-424.

Verbruggen, F., and Logan, G. D. (2009). Models of response inhibition in the stop-signal and stop-change paradigms. Neurosci. Biobehav. Rev. 33, 647-661.
Warren, L. R., and Morton, J. (1982). The effects of priming on picture recognition. Br. J. Psychol. 57, 147-154.

Xue, G., Aron, A. R., and Poldrack, R. A. (2008). Common neural substrates for inhibition of spoken and manual responses. Cereb. Cortex 18, 1923-1932.

Conflict of Interest Statement: The authors declare that the research was conducted in the absence of any commercial or financial relationships that could be construed as a potential conflict of interest.

Received: 04 March 2010; paper pending published: 25 March 2010; accepted: 10 August 2010; published online: 29 September 2010.

Citation: van den Wildenberg WPM and Christoffels IK (2010) STOP TALKING! Inhibition of speech is affected by word frequency and dysfunctional impulsivity. Front. Psychology 1:145. doi:10.3389/ fpsyg.2010.00145

This article was submitted to Frontiers in Cognition, a specialty of Frontiers in Psychology.

Copyright $($ C) 2010 van den Wildenberg and Christoffels. This is an open-access article subject to an exclusive license agreement between the authors and the Frontiers Research Foundation, which permits unrestricted use, distribution, and reproduction in any medium, provided the original authors and source are credited. 